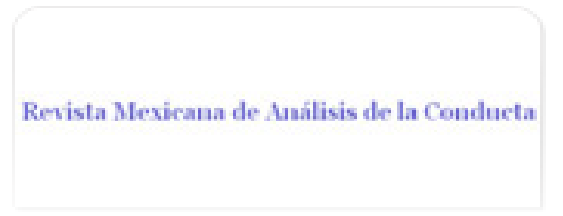

Revista Mexicana de Análisis de la Conducta ISSN: 0185-4534

editora@rmac-mx.org

Sociedad Mexicana de Análisis de la Conducta México

Arroyo Hernández, Rosalinda; Mares Cárdenas, Guadalupe

Efectos del tipo funcional de entrenamiento sobre el ajuste lector

Revista Mexicana de Análisis de la Conducta, vol. 35, núm. 1, junio, 2009, pp. 19-35

Sociedad Mexicana de Análisis de la Conducta

Guadalajara, México

Disponible en: http://www.redalyc.org/articulo.oa?id=59311416002

- Cómo citar el artículo

- Número completo

- Más información del artículo

- Página de la revista en redalyc.org

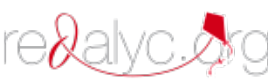

Sistema de Información Científica

Red de Revistas Científicas de América Latina, el Caribe, España y Portugal Proyecto académico sin fines de lucro, desarrollado bajo la iniciativa de acceso abierto 


\title{
EFECTOS DEL TIPO FUNCIONAL DE ENTRENAMIENTO SOBRE EL AJUSTE LECTOR
}

\author{
EFFECTS OF THE FUNCTIONAL TYPE OF TRAINING \\ ON THE READING ADJUSTMENT \\ ROSALINDA ARROYO HERNÁNDEZ \\ Y GUADALUPE MARES CÁRDENAS ${ }^{1}$ \\ UNIVERSIDAD NACIONAL AUTÓNOMA DE MÉXICO- \\ FACULTAD DE ESTUDIOS SUPERIORES IZTACALA
}

\begin{abstract}
RESUMEN
El presente trabajo tuvo por objetivo evaluar los efectos de entrenar cinco tipos de contacto con las relaciones entre objetos y sus elaboraciones lingüísticas sobre el ajuste lector de estudiantes universitarios. Primeramente los participantes fueron expuestos a una evaluación de ajuste lector, consistente en preguntas de diferente complejidad funcional, para posteriormente ser asignados aleatoriamente a uno de cinco grupos experimentales diferenciados por el nivel de complejidad funcional del entrenamiento (Contextual, Suplementaria, Selectora, Sustitutiva Referencial y Sustitutiva No Referencial), un sexto grupo (Control) no tuvo entrenamiento alguno. Este entrenamiento fue realizado para establecer relaciones entre el objeto (proyectiles en movimiento) y expresiones lingüísticas, variando entre los grupos la complejidad de estas relaciones. Posteriormente los seis grupos fueron expuestos a una segunda evaluación en ajuste lector. Los resultados demuestran que conforme se incrementa la complejidad funcional de las relaciones objeto-expresión lingüística aumentan el porcentaje promedio de aciertos, específicamente en
\end{abstract}

1. Este estudio se realizó como parte de la Tesis Doctoral de la primera autora bajo la asesoría de la segunda autora. La correspondencia sobre este artículo deberá enviarse a Rosalinda Arroyo Hernández, Ave. de los Barrios \# 1, Los Reyes Iztacala, Tlalnepantla, Edo. Mex., México o al correo electrónico: rarroyo@campus.iztacala.unam.mx

Recibido 13 de Noviembre de 2008. Revisado: 7 de enero de 2009. Aceptado: 3 de febrero de 2009. 
los grupos Sustitutivos. Los resultados se discuten a la luz de los factores que modulan la probabilidad de la estructuración del comportamiento en niveles complejos.

Palabras clave: Niveles funcionales, ajuste lector, criterio de ajuste.

\begin{abstract}
This study evaluated the effects of training five types of contact with the relations between objects and their linguistic elaborations on reading adjustment performance of University students. The participants were exposed to an evaluation of reading adjustment, consistent of questions of different functional complexity, they were later assigned to one of five experimental groups differentiated by the level of functional complexity of the training (Contextual, Supplementary, Selective, Referential Substitutive and Non-Referential Substitutive), a sixth group (Control) did not have any training. This training was done to establish relations between the object (projectiles in movement) and linguistic expressions, the complexity of these relations changed between groups. Later all groups were exposed to the second reading adjustment evaluation. Results show that as the functional complexity of the object- linguistic expression relations increased the average percentage of correct responses increased, specifically in the Substitutives groups. Results are discussed emphasizing the factors that determine the probability of the structuring of behavior in complex levels.
\end{abstract}

Key words: Functional level, reading adjustment, adjustment criteria.

En las exploraciones empíricas desarrolladas desde la tradición que define a la psicología como una ciencia de la conducta se ha seguido la estrategia de "crear historias controladas" con el fin de investigar y explicar lo que un organismo hace en el presente. En el Análisis Experimental de la Conducta y en los trabajos pioneros de Skinner et al. (Skinner, 1938; 1972; Ferster \& Skinner, 1957) la creación experimental de dichas "historias" se conceptuó como "historia de condicionamiento", buscándose la exploración de los parámetros involucrados en la relaciones de contingencia denominadas respondiente y operante.

En la década de los ochenta se elaboraron clasificaciones de la conducta adicionales a la distinción operante/respondiente, como las propuestas por Sidman (1986) o por Ribes y López (1985). Cada una de estas nuevas clasificaciones llevó a la creación de historias controladas que indagaran los efectos de los parámetros particulares implicados en cada una de ellas. Por ejemplo, los trabajos realizados bajo el paradigma de relaciones de equiva- 
lencia de Sidman se han ocupado de estudiar el tamaño de las clases de equivalencia, o el número de nódulos y su distancia asociativa (Fields \& Verhave, 1987).

Por su parte, los trabajos articulados con la clasificación de la conducta en términos de los niveles funcionales formulados por Ribes y López se han dirigido a evaluar parámetros como los procesos de estructuración temporal en niveles contextual, suplementario y selector (Carpio, et al., 2001), el papel de las descripciones lingüísticas en el tránsito del nivel selector al sustitutivo referencial (Moreno, Cepeda, Tena, Hickman \& Plancarte, 2005) o la transferencia de conductas relacionales al modificar el nivel funcional de entrenamiento (Mares, Guevara \& Rueda, 1996; Mares, Ribes \& Rueda, 1993).

La investigación aquí presentada se ubica en la perspectiva de campo postulada por Kantor $(1933 ; 1959)$ y en la clasificación de los niveles funcionales ya mencionada. En este trabajo estudiaremos el efecto de entrenar diferentes niveles funcionales de contacto con las relaciones entre objetos y sus elaboraciones lingüísticas sobre el ajuste lector en universitarios. A continuación aclararemos las variables involucradas en esta investigación.

Los niveles funcionales propuestos por Ribes y López (1985) describen relaciones de contingencia de diferente complejidad, a saber: Contextual, en el cual la participación del sujeto no puede alterar la constancia en las relaciones presentes en el ambiente, Suplementario aquel en el cual la respuesta del organismo es capaz de modificar las relaciones contingenciales presentes, Selector en el cual las relaciones de contingencialidad cambian momento a momento en función de un evento ajeno que se agrega a las relaciones ya existentes, Sustitutivo Referencial en el que lingüísticamente se media el contacto con las relaciones de contingencia lo que permite separarse de la situacionalidad o contigüidad temporal y Sustitutivo no Referencial en el cual las relaciones de contingencia ya no dependen de la presencia o incluso existencia de los objetos de estímulo, de modo que las relaciones de contingencia se establecen con sustitutos verbales abstractos.

Carpio et al. (Carpio, Arroyo, Canales, Flores \& Morales, 2000; Arroyo, et al., 2005; Morales, et al., 2005) desarrollaron el concepto de Ajuste Lector con el fin de abarcar conceptualmente, desde una aproximación Interconductual, los fenómenos que se describen bajo los términos comprensión de textos o comprensión lectora. El ajuste lector se define como la correspondencia funcionalmente pertinente del lector a la situación de lectura y a los criterios que ésta impone. Esta caracterización del ajuste lector implica la identificación de los criterios de correspondencia funcionalmente pertinentes. Para ello tanto Carpio (1994) como Ribes, Moreno y Padilla (1996) reconocen cinco tipos generales de criterios progresivamente más complejos e inclusivos, que se relacionan, más no se igualan, con los niveles de aptitud funcional propuestos por Ribes y López (1985), teniéndose así criterios de ajustividad 
(en Carpio) o diferenciación (en Ribes et al.), de efectividad, de pertinencia (en Carpio) o precisión (en Ribes et al.), de congruencia y de coherencia. Con base en estos criterios es posible realizar análisis finos del ajuste lector.

Los primeros trabajos empíricos que conceptuaron la creación de historias en términos de "niveles funcionales de desligamiento", diseñando preparaciones experimentales que permitieran estudiarlas, son las realizadas por Mares et al. (Mares, 1988; Mares, Guevara \& Rueda, 1996; Mares, Ribes \& Rueda, 1993; Mares, Rueda \& Plancarte, 1994). Ellos estudiaron el efecto de instruir la elaboración de relaciones lingüísticas -entrenando diferentes niveles de contacto de niños con las relaciones entre objetos y sus elaboraciones lingüísticas-, sobre la transferencia de lo aprendido.

Las investigaciones anteriores y trabajos posteriores (Bazán \& Mares, 2002; Mares, Rivas \& Bazán, 2002) aportaron evidencia que demuestra la factibilidad de realizar entrenamientos específicos con base en los niveles funcionales e indican que el nivel funcional en el cual se instruye a elaborar lingüísticamente una relación, afecta el grado de transferencia de la expresión relacional hacia otros eventos susceptibles de ser elaborados de la misma manera. En particular, Mares et al. encontraron que el nivel funcional en el cual se aprende y ejercita una competencia lingüística promueve diferencias considerables a favor del grupo más complejo, lo cual sugiere que el nivel funcional en que se entrene o ejercite una competencia funge como un factor disposicional de la ejecución futura.

Si bien los estudios previamente citados demuestran que el entrenamiento en niveles Sustitutivos genera una mejor ejecución en tareas de naturaleza funcionalmente semejante, otras investigaciones han fallado en hallar este resultado, incluso encuentran exactamente lo contrario, es decir que conforme se incrementa la complejidad funcional el desempeño va disminuyendo, un ejemplo de este resultado es el estudio de Fuentes y Ribes (2006) quienes con el objetivo de evaluar la influencia del conocimiento temático en la lectura, expusieron a niños a contactos mediados lingüísticamente con el referente del texto. Para ello, un primer grupo observó un documental previo a la lectura, el segundo también fue expuesto al mismo documental pero además durante éste se instaba a los participantes para que describieran lo que habían visto y un tercer grupo no recibió ningún entrenamiento. Posteriormente los tres grupos leían el mismo texto y fueron evaluados en cinco tareas diferentes que imponían criterios de diferente complejidad funcional. Los autores encontraron en ambos grupos experimentales decrementos en los porcentajes de aciertos conforme se incrementó la complejidad funcional de las pruebas, en cambio el grupo control presentó un mejor desempeño tanto global como en las preguntas más complejas, de modo que pareciera que tanto la proyección del documental como la elaboración lingüística posterior no sólo no promovieron ajustes eficaces sino que los entorpecieron. 
Un estudio en el que las descripciones lingüísticas no parecen promover ajustes complejos es el de Arroyo et al. (2005) en el que se evaluó los efectos de entrenar distintos tipos de contactos previos con el referente de un texto en una prueba de ajuste lector. Se tuvieron cinco grupos experimentales, correspondientes a cada nivel funcional y se encontró que el porcentaje de respuestas correctas, en la prueba de ajuste lector, fue incrementándose ligeramente conforme se acrecentaba el nivel de complejidad del entrenamiento, lo cual parece semejante a lo encontrado por Mares et al. No obstante las diferencias entre los grupos son pocas e incluso el grupo Sustitutivo No Referencial obtiene resultados ligeramente inferiores a los del grupo adyacente, el Sustitutivo Referencial.

En breve, algunos estudios demuestran que entrenar en niveles funcionales complejos genera una mejor ejecución en pruebas de transferencia, mientras que otros encuentran exactamente lo contrario, esto es interesante pues teóricamente esperaríamos que entrenamientos en un nivel funcional particular generase coincidencias más claras. Por ello, es necesario preguntarnos si la falta de diferenciación entre los niveles reportada en los estudios citados obedezca a cuestiones metodológicas, es decir, que las tareas que fueron planeadas para promover un cierto nivel funcional no sean las más adecuadas.

Un elemento común en los trabajos que reportan desempeños bajos en los grupos con entrenamiento complejo es que las tareas son lectura de textos o exposiciones verbales diferenciándose entre ellos por el grado de especificidad como si el factor lingüístico fuese suficiente para promover el establecimiento del comportamiento en niveles complejos. Mientras que los trabajos en los cuales se ha encontrado que los niveles más complejos (sustitutivos) aumentan la probabilidad de transferencia, se caracterizan por incluir, además de las expresiones lingüísticas relacionales, el contacto con los eventos ante los cuales la expresión relacional cobra sentido. Por ejemplo Mares, Rivas y Bazán (2002) evaluaron el efecto que tiene el nivel funcional de entrenamiento en las transferencias de competencias en el modo oral al modo escrito. Para lo cual cuatro grupos de niños fueron expuestos a tareas de diferente complejidad funcional: El grupo Contextual copiaba textos, el grupo Suplementario alteraba y corregía un texto con base en otro, el grupo Selector debía seleccionar de un texto la respuesta pertinente para una pregunta literal, finalmente el grupo Sustitutivo Referencial debía describir dibujos y ordenarlos secuencialmente con base en una historia leída anteriormente, ésto se realizó durante 10 sesiones. Los resultados encontrados demostraron efectos del nivel funcional de entrenamiento sobre la probabilidad de expresar relaciones al escribir sobre temas no entrenados, encontrando el menor incremento en el grupo contextual y el mayor incremento en el grupo sustitutivo. Estos resultados apoyan la suposición de que el nivel funcional en 
que se entrene o ejercite una competencia funge como un factor disposicional que modula jerárquicamente la ejecución.

De hecho, después de realizar varias investigaciones Mares, et al. (1996) concluye que para lograr la estructuración de interacciones sustitutivas referenciales, en niños de primaria, se requiere que el niño establezca un contacto cuasi simultáneo entre la expresión lingüística relacional y el evento pertinente (los objetos que se relacionan) o dibujos que sustituyan la relación entre ellos (secuencias de imágenes, videos o representaciones).

Aparentemente, el factor lingüístico no es suficiente para promover el desarrollo de interacciones sustitutivas no referenciales, sino que se requiere también del contacto con el evento ante el cual la expresión lingüística relacional es pertinente, de modo que se requiere mayor investigación sobre los parámetros necesarios para que la elaboración lingüística de los eventos de estímulo se establezcan en los niveles complejos. Por ello, en esta investigación estudiaremos, el efecto de entrenar cinco tipos de contacto de estudiantes con las relaciones entre objetos y sus elaboraciones lingüísticas, sobre el ajuste lector.

\section{MÉTODO}

\section{Participantes}

Participaron voluntariamente 60 estudiantes de primer año de la carrera de psicología de la FES Iztacala-UNAM, todos del sexo femenino y del turno matutino, los cuales fueron seleccionados mediante su desempeño en la evaluación inicial en ajuste lector. El criterio de ejecución impuesto fue la obtención de menos de 41 aciertos (59.4\%).

\section{Materiales e instrumentos}

El texto que se empleó trató sobre el movimiento y las dos primeras leyes de Newton, con un total de 1094 palabras, distribuidas en 18 párrafos, letra arial, 12 puntos. El cuestionario estuvo constituido por 69 preguntas, 12 de estas preguntas en la forma de enunciados incompletos (todas pertenecientes al criterio de Efectividad) y 57 preguntas de opción múltiple, con cuatro opciones de respuesta, de las cuales sólo una era correcta.

Un ejemplo ${ }^{2}$ de una pregunta de Ajustividad es: ¿Para qué se crearon los conceptos de fuerza y energía?, R.- Para analizar y predecir la naturaleza de

2. Por cuestiones de espacio, solo presentamos ejemplos de las preguntas y de la opción de respuesta correcta, si el lector requiriese los materiales íntegros puede solicitarlos al correo de contacto. 
los movimientos" (15 preguntas). Del de Efectividad: Para que un objeto presente cambios en su aceleración debe entrar en contacto con otro $(12$ preguntas); Del de Pertinencia: Manteniendo la fuerza idéntica ¿Cómo sería la aceleración de un objeto que tuviese una masa mayor con respecto a uno que tuviese una masa menor? R.- La aceleración sería menor" (15 preguntas); Del de Congruencia: "Si tienes un carro de juguete que se mueve a una velocidad mayor que otro y ambos han sido lanzados con la misma fuerza y al mismo tiempo, se dice que es por: R.- El principio de la masa inercial de los objetos" (14 preguntas); Del de Coherencia: “ ¿Cuál de las siguientes fórmulas sería adecuada para describir la aceleración?, R.- $A=V / T$, donde $A$ es aceleración, $\mathrm{V}$ velocidad y T tiempo" (13 preguntas).

\section{Aparatos}

La programación y registro de eventos se realizó mediante sistemas de cómputo Pentium-IV, empleando un programa diseñado con el ambiente de programación Visual Basic 6.0 y aplicaciones de Macromedia Flash MX, ambos compatibles con la plataforma Windows XP, el cual presentó cinco diferentes subrutinas dependiendo del entrenamiento.

\section{Diseño}

Se empleó un diseño de grupo control-experimentales pretest-postest (Kerlinger \& Lee, 2002). En este diseño los participantes son asignados aleatoriamente (sin reemplazo) a uno de seis grupos: un grupo control y cinco experimentales, éstos diferenciados por el nivel funcional de entrenamiento (Contextual, Suplementario, Selector, Sustitutivo Referencial y Sustitutivo no Referencial) y en el que todos los grupos pasan por ambas evaluaciones. La elección del diseño obedeció a la necesidad de seleccionar participantes con desempeños bajos iniciales y el interés por hacer comparaciones entre grupos e intra grupo entre ambas pruebas. Los posibles efectos de la administración repetida de la prueba fueron controlados con el grupo sin entrenamiento.

\section{Procedimiento}

Fase 1: Evaluación 1. Como ya se mencionó esta evaluación fue la que sirvió para seleccionar a los participantes, la cual inició entregando el texto y dándoles de forma oral la instrucción de que leyeran el texto cuidadosamente pues después se les harían preguntas sobre lo que leyeron. Conforme los participantes declaraban haber terminado de leer el texto, se retiraba éste y se hacía entrega del cuestionario, aleatorizado tanto en términos del tipo de preguntas como de la ubicación de la respuesta correcta en el caso de las preguntas de opción múltiple. Esta fase tuvo una duración promedio de una hora y se realizó, a papel y lápiz, en los salones de clase de los participantes. 
Fase 2: Entrenamiento. Los participantes seleccionados fueron asignados aleatoriamente a uno de seis grupos, cinco experimentales y uno control. Cada grupo experimental recibió un entrenamiento distinto, diseñado para promover tipos diferenciales de contacto con el referente del texto de prueba. El grupo Control no recibió entrenamiento alguno y fue expuesto directamente a la segunda prueba de ajuste lector.

La tarea fue presentada mediante sistemas de cómputo, emulando un juego de video, cuyo objetivo fue ganar puntos, el modo de ganar los puntos en cada "juego" fue diferente dependiendo del nivel de complejidad del entrenamiento. Independientemente del tipo de entrenamiento cada sesión estuvo constituida por 10 ensayos y el criterio para terminar con esta fase fue la obtención del $80 \%$ de aciertos (mínimo de ocho puntos), durante tres sesiones consecutivas. El tratamiento particular para cada grupo se detalla a continuación:

a) Al grupo Historia Contextual se le presentó en la pantalla del monitor dos muñecos cada uno con su propia plataforma (una mesa) y proyectil (una bola de metal), el participante debía oprimir, mediante el mouse de la computadora, un botón para que la bola rodara sobre la plataforma. Las trayectorias seguidas fueron idénticas o diferentes, dependiendo de tres propiedades: el tamaño de la bola (masa), la altura de la mesa (gravedad) y la velocidad del tiro (fuerza). Estas propiedades variaban ensayo a ensayo pero en cada uno sólo se alteraba una de dichas propiedades manteniendo constantes las otras. Una vez que el participante había observado los dos tiros se habilitaba una sección inferior de la pantalla en la que aparecía la pregunta "los tiros fueron iguales o diferentes" y la obtención del punto dependía de la identificación correcta del parecido entre los tiros. Así, este entrenamiento promovió que el contacto con el referente se limitara a propiedades invariantes en tiempo y espacio, de modo que la respuesta del participante en ningún caso podía alterar la ocurrencia de los eventos, sólo ajustar su respuesta diferencialmente a ellos (en este caso a si eran parecidos o no los tiros).

b) En el grupo Historia Suplementaria se pidió al participante "tirar" la bola desde la plataforma hacia una meta, delimitada por dos líneas rojas de modo tal que la bola no rebasará la segunda línea roja. En la pantalla se presentó una bola inmóvil sobre una mesa así como dos opciones que podían afectar el tiro. Dependiendo de la opción elegida la bola seguía cierta trayectoria y rebasaba o no la meta. En cada ensayo se cambiaban las opciones en función de fuerza, masa o velocidad. La obtención del punto dependía de la elección correcta de la propiedad que permitiera que la bola llegara lo más cerca de la segunda línea sin rebasarla. Por ejemplo si debía optar entre tirar con una mesa grande o pequeña 
con una bola también pequeña la elección de esta última fue correcta. Así, en este entrenamiento el contacto con el referente permitía que el participante modificara la ocurrencia de los eventos (al elegir una u otra propiedad del tiro), posibilitándose la afectación directa de la respuesta del participante sobre el ambiente (que la bola rebasará, o no, la meta).

c) Para el grupo Historia Selectora la tarea consistió en elegir una opción que permitiera que su tiro no rebasase la línea pero ahora las elección no solo estaba en función de alguna de las tres propiedades sino del tiro de un jugador contrario (virtual). Para ello, se presentaba en la parte superior de la pantalla un jugador tirando una bola, el participante debía observar ese tiro y elegir entre tres posibles elecciones, presentados en la parte inferior, una vez que el participante elegía se presentaba una nueva pantalla en la que podía observar si efectivamente su tiro fue mejor que el del oponente. En cada ensayo se modificaba alguna de las tres propiedades y la obtención del punto dependía de elegir la variación correcta en función de lo observado en el tiro del otro jugador. Así, el contacto con el referente se establecía en términos no de las propiedades aparentes o temporales de los eventos, sino de relaciones condicionales entre éstos, relaciones que varían constantemente, de modo que para que el participante pueda seguir siendo efectivo su respuesta debía ajustarse diferencialmente a la variabilidad del ambiente.

d) Al grupo Historia Sustitutiva Referencial se le indicaba que debía ayudar a un compañero -también virtual- a ganarle a otro jugador y para ello debía observar el tiro del contrario y después "decirle" (escribiéndolo en cajas de texto) a su compañero el modo en que debe tirar o el tamaño de la mesa o bola que debía usar. Así en la pantalla se mostraba en la parte superior un jugador tirando una bola y cuando terminaba el tiro, la imagen desaparecía y en su lugar se presentaba una oración que describía lo que había hecho el jugador contrario así como la imagen del compañero y tres cajas de texto, en la cual (dependiendo de su elección) debía escribir su instrucción, una vez escrita la instrucción se presentaba otra pantalla en la cual se podía observar el tiro elegido. En esta ocasión la obtención del punto dependía de que a partir de las contingencias sustituidas en la oración se eligiera la opción correcta y a su vez fuese congruente con la instrucción que los participantes daban a su "compañero". Por tanto, en este entrenamiento el contacto con el referente se establecía mediante la descripción de eventos no presentes en tiempo y espacio pero con referencia empírica concreta (la descripción de los tiros y la instrucción al "compañero" estaba siempre delimitada por propiedades específicas).

e) Al grupo Historia Sustitutiva No Referencial se le presentó en la parte superior de la pantalla dos jugadores cada uno con su respectiva mesa y bola, cuando el participante observó ambos tiros se presentó un enun- 
ciado sobre un principio del movimiento de los objetos y tres opciones de respuesta (también enunciados), solo una de ellas se relacionaba correctamente con el enunciado anterior. Así, la obtención del punto era resultado de la coherencia entre dos segmentos lingüísticos (la oración y la opción de respuesta). En este entrenamiento el contacto con el referente se establecía a partir de propiedades convencionales mediadas por el lenguaje de una disciplina particular, promoviéndose que la respuesta lingüística del participante al mediar su propio comportamiento también en términos lingüísticos y abstractos, reorganice las relaciones convencionales.

Fase 3: Evaluación 2. Una vez concluida la fase de entrenamiento, todos los grupos (tanto control como experimentales) realizaron la segunda evaluación, la cual fue idéntica a la primera, excepto que tanto el orden de presentación de las preguntas como la posición de la opción correcta era diferente.

\section{RESULTADOS}

En la figura 1 se presenta el porcentaje promedio de aciertos totales en ambas evaluaciones, y se puede observar que en la primera evaluación (barras blancas) todos los grupos presentan ejecuciones muy similares lo que permite asumir que los niveles de entrada son semejantes, conjetura corroborada por el análisis estadístico $\left(\mathrm{F}_{(5,59)}=0.368, \mathrm{p}=0.868\right)$ al no obtenerse diferencias significativas entre ellos. Estos resultados confirman que el criterio de ejecución fue efectivo para igualar el nivel de entrada de los grupos, lo que a su vez permite asegurar que los posibles cambios posteriores al entrenamiento no fuesen debidos a diferencias iniciales entre grupos. 
Por tipo de Entrenamiento

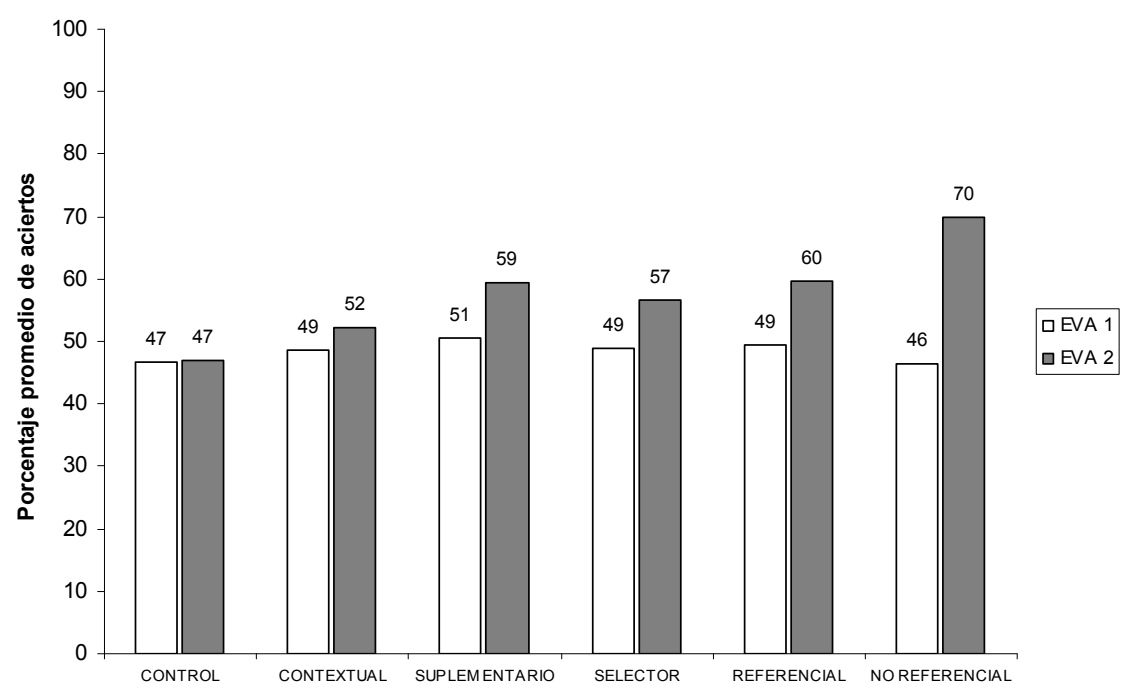

Figura 1. Porcentajes promedio de aciertos obtenidos por cada grupo en ambas evaluaciones.

En la misma figura se presenta los porcentajes obtenidos durante la evaluación 2 (barras grises), pudiéndose apreciar una función positiva de la complejidad de la historia, esto es, mayor porcentaje de aciertos a medida que se incrementa la complejidad funcional del entrenamiento, con excepción de grupo Selector. El análisis estadístico correspondiente demuestra un efecto significativo de grupo $(F(5,59)=10.827, p<0.001)$, la prueba post hoc demuestra diferencias entre el grupo Control y cuatro de los grupos experimentales, con excepción del grupo Contextual, éste a su vez, es diferente de todos los grupos salvo el Control y Selector; de modo paralelo los grupos Suplementario, Selector y Referencial obtienen diferencias con los grupos más alejados, no así con los adyacentes, finalmente, la ejecución del grupo Sustitutivo No Referencial es diferente significativamente de todos los grupos, tanto control como experimentales.

A partir de estos resultados se puede afirmar que los diferentes tipos de entrenamiento generaron diferencias en el ajuste lector, y además que a medida que se incrementa la complejidad del entrenamiento, el ajuste lector es afectado positivamente permitiendo la posibilidad de responder adecuadamente un mayor porcentaje de preguntas. 
Este análisis es confirmado al comparar la mejoría en la segunda evaluación con respecto a la primera (ver figura 1), pudiéndose apreciar que el grupo Control prácticamente no presenta mejoría alguna, que sólo los grupos Experimentales incrementan su porcentaje de aciertos y que dicho incremento está directamente relacionado con la complejidad funcional del entrenamiento.

Para conocer si los incrementos de la primera evaluación a la segunda son significativos en cada grupo se realizaron pruebas t para muestras relacionadas (ver tabla 2), encontrándose que excepto por los grupos Control y Contextual, en todos los grupos el porcentaje de aciertos mejora significativamente (Suplementario: $\mathrm{t}_{(9)}=3.765, \mathrm{p}=0.004$, Selector: $\mathrm{t}_{(9)}=4.261, \mathrm{p}=0.002$, Sustitutivo Referencial: $\mathrm{t}_{(9)}=3.143, \mathrm{p}=0.012$, Sustitutivo No Referencial: $\mathrm{t}_{(9)}=$ 4.800, $p=0.001$ )

En breve, los resultados hasta ahora presentados indican que existen diferencias entre recibir el entrenamiento con respecto a no recibirlo y además que los efectos observados en la segunda evaluación (mejoría en el porcentaje de aciertos obtenidos en la prueba de ajuste lector) son diferenciales en términos del tipo funcional del entrenamiento.

Un análisis más puntual implica la exploración de las posibles diferencias de los efectos de cada entrenamiento sobre cada tipo de pregunta, para lo cual en la figura 2 se presenta el porcentaje de aciertos en ambas evaluaciones para todos los grupos en cada uno de los tipos de preguntas. 


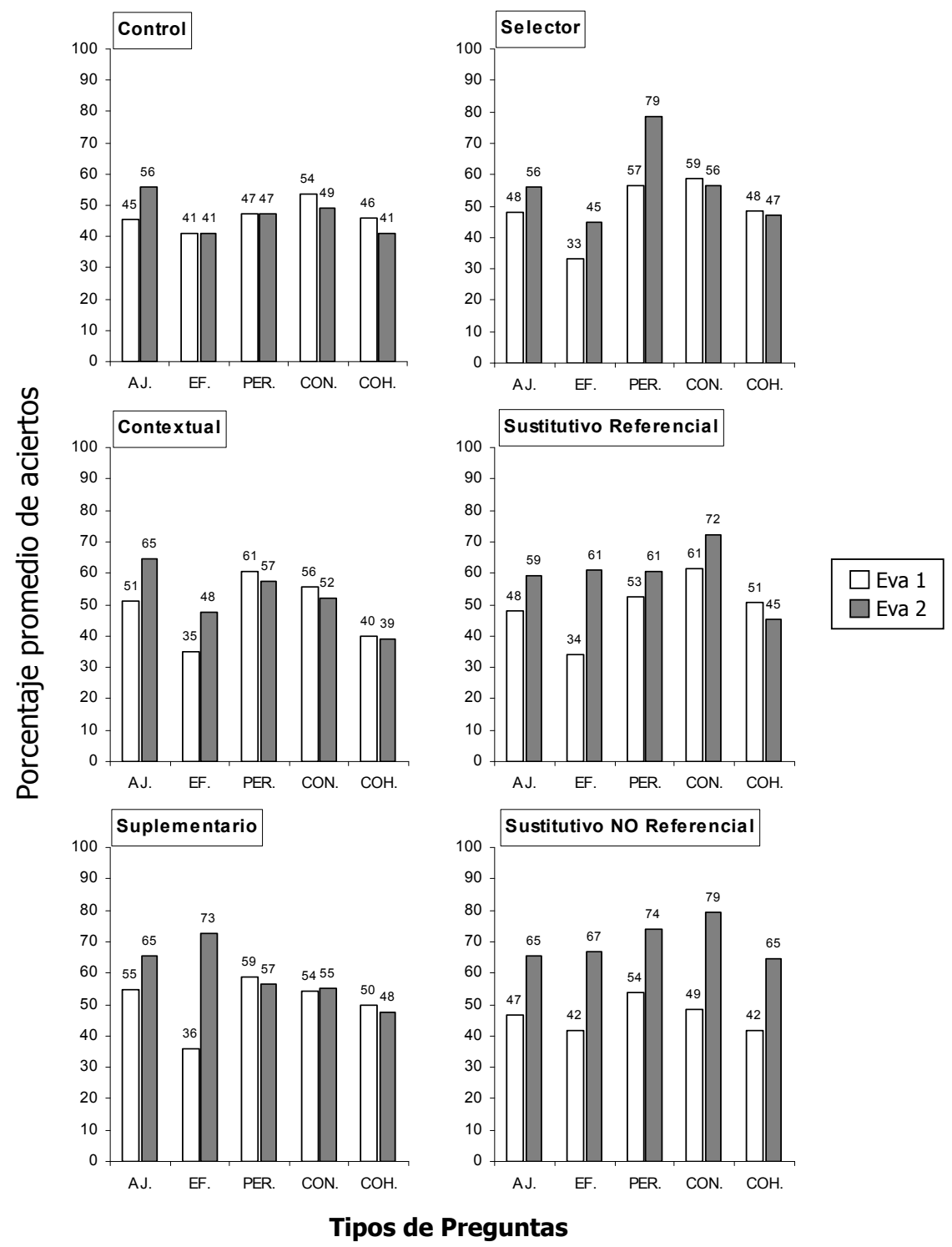

Figura 2. Porcentajes promedios de aciertos ante cada tipo de pregunta para cada grupo en ambas evaluaciones. 
En dicha figura se muestra que el grupo Control sólo mejora en las preguntas más simples, las elaboradas bajo el criterio de ajustividad, manteniéndose igual en efectividad y pertinencia, pero con decrementos en las preguntas con los criterios más complejos, congruencia y coherencia. Una función similar se observa en el grupo Contextual, excepto porque este grupo también presenta mejoría en las preguntas de efectividad. De manera paralela al grupo anterior, el grupo Suplementario exhibe incrementos en las preguntas de ajustividad y efectividad.

En el caso del grupo Selector se muestran aumentos en el porcentaje de aciertos en los primeros tres tipos de preguntas, hallándose el mayor incremento en las preguntas de pertinencia, no así en las preguntas más complejas, en las cuales incluso se advierten ligeros decrementos. Este dato es acorde con lo obtenido con los grupos previos, en el sentido de que los incrementos durante la segunda evaluación en ningún caso se aprecian ante las preguntas más complejas (congruencia y coherencia)

Para el grupo Sustitutivo Referencial se presentan mayores porcentajes de aciertos en la segunda evaluación en ajustividad, efectividad, pertinencia y ahora también en las preguntas de congruencia. Finalmente para el grupo Sustitutivo No Referencial se observa mejoría en todos los tipos de preguntas.

\section{DISCUSIÓN}

El presente trabajo tuvo como propósito general evaluar los efectos del nivel funcional de entrenamiento sobre el desempeño de universitarios en una prueba de ajuste lector. Para lo cual se construyeron experimentalmente cinco tipos de relaciones entre los objetos de referencia y sus elaboraciones lingüísticas (grupos experimentales), relaciones que iban de lo más simple a lo más complejo, mientras que en un sexto grupo no tuvo ningún tipo de entrenamiento (grupo control).

Con ello se pretendía, inicialmente, demostrar la factibilidad de entrenar relaciones entre los objetos y sus elaboraciones lingüísticas en distintos niveles de complejidad y observar si ésto afecta diferencialmente el ajuste lector posterior, por tanto lo que se esperaría es que el grupo sin entrenamiento, en el que no se construyeron dichas relaciones, tenga un desempeño inferior al de aquellos grupos en los que si se establecieron (independientemente de la cualidad de éste). Tal contrastación, precisamente demuestra que el grupo Control tuvo un desempeño inferior al de los grupos con entrenamiento.

Un segundo punto de interés es que tales relaciones pueden distinguirse en términos de la complejidad funcional del contacto. Al respecto, los datos del presente estudio indican efectos diferenciales en el ajuste lector, siendo éste más eficiente conforme se incrementa la complejidad de dicho contac- 
to. Este resultado difiere de lo hallado previamente por Arroyo et al. (2005) quienes si bien encuentran una función similar hasta el grupo Sustitutivo Referencial, dicha función se detiene en el grupo más complejo (Sustitutivo no Referencial) presentando desempeños semejantes a los del grupo Selector. A diferencia, lo hallado en este trabajo demuestra la posibilidad de promover ajustes en niveles más complejos y sugieren que es necesario examinar con más detalle la naturaleza de las tareas empleadas.

Un análisis más fino implica preguntarnos si existe relación entre el nivel funcional de entrenamiento y los diferentes tipos de preguntas que evalúan el ajuste lector, encontrándose la obtención de mayores porcentajes de aciertos conforme se incrementa la complejidad del entrenamiento, de modo tal que el grupo con entrenamiento más complejo presenta mayores porcentajes de aciertos pues fue capaz de responder correctamente preguntas de todos los criterios, mientras que el grupo que recibió el entrenamiento más simple, funcionalmente hablando, al sólo poder responder las preguntas de los criterios más sencillos obtiene menores porcentajes de aciertos. Estos resultados apuntan a que el alcance del entrenamiento no se restringe a efectos positivos en el incremento de aciertos generales sino que tales incrementos están vinculados con el tipo funcional de criterio bajo el cual se elaboraron las preguntas.

Lo encontrado con los grupos Sustitutivos es particularmente importante pues estos grupos fueron expuestos, al igual que los otros, a la observación del movimiento de los proyectiles con la diferencia de que en éstos el objeto estaba vinculado a expresiones lingüísticas de diferente abstracción. Al parecer esta asociación entre el objeto referente y la expresión relacional promueve que el lector sea capaz de estructurar su comportamiento en los niveles complejos. Este dato es soportado por evidencia de Ibáñez y Reyes (2002) quienes al exponer a universitarios a la presencia o ausencia del referente de un texto demuestran que la ausencia del objeto de referencia afecta negativamente la ejecución en la identificación de elementos del objeto real, resultando especialmente relevante que la mera presencia de dicho objeto tampoco garantiza mejores resultados de modo que parece que la presencia del objeto debe estar acompañada de la descripción verbal.

Por ello, la falta de correspondencia de lo encontrado en el presente trabajo con lo obtenido en trabajos previos (Arroyo et al., 2005) con respecto al grupo Sustitutivo no Referencial puede obedecer a que en el estudio precitado el entrenamiento se centró en la mera verbalización lingüística aislada del contacto directo con el referente.

De hecho, en los estudios realizados por Mares et al. la manera de elaborar lingüísticamente las relaciones entre objetos se mantuvo constante, no obstante, Mares (2000) afirmó que el grado de transferencia se ve también afectado por la naturaleza de la expresión relacional, de tal manera que la 
expresiones abstractas promoverían más transferencia porque se pueden aplicar, de manera pertinente, a más fenómenos. En cambio en este estudio, las relaciones entre los objetos se mantienen constantes, mientras que el grado de abstracción de la expresión relacional se modifica con el fin de establecer los ejercicios de instrucción en el nivel funcional que se pretende dar el entrenamiento. Por tanto parece que la promoción de niveles sustitutivos no referenciales requiere como uno de sus elementos la vinculación entre las relaciones entre objetos y las expresiones relacionales tanto concretas como abstractas. Futuras investigaciones serán dirigidas a corroborar tal hipótesis.

\section{REFERENCIAS}

Arroyo, R., Morales, G., Pichardo, A., Canales, C., Silva, H. \& Carpio, C. (2005). ¿Cómo se aprende a comprender?: Análisis funcional de la historia con los referentes. En C. Carpio y J. J. Irigoyen (Eds.). Psicología y Educación: Aportaciones desde la teoría de la conducta. UNAM: FES Iztacala.

Bazán, A. \& Mares, G. (2002). Influencia del nivel funcional de entrenamiento en la elaboración relacional en tareas de ejecución verbal. Revista Mexicana de Análisis de la Conducta, 28(1), 19-40.

Carpio, C. (1994). Comportamiento animal y teoría de la conducta. En: L., Hayes, E., Ribes y F., López (Eds.). Psicología Interconductual. México: EDUG.

Carpio, C., Flores, C., Bautista, E., González, F., Pacheco, V., Páez, A. \& Canales, C. (2001). Análisis experimental de las funciones contextual y selectora. En: G. Mares \& Y. Guevara. (Coords.). Psicología Interconductual. Avances en investigación básica (pp. 9-36). México, UNAM, FES Iztacala - DGAPA.

Carpio, C., Arroyo, R., Canales, C., Flores C. \& Morales G. (2000). Un modelo de análisis de la comprensión de textos. Quinto Congreso Internacional sobre Conductismo y Ciencias de la Conducta, Xalapa, Veracruz, México, octubre.

Ferster, C., B. \& Skinner, B., F. (1957). Schedules of reinforcement. Englewood cliffs: Prentice Hall

Fields, L., \& Verhave, T. (1987). The structure of equivalence classes. Journal of the Experimental Analysis of Behavior, 48, 317-332.

Fuentes, T. \& Ribes, E. (2006). Influencia de tres repertorios precurrentes en la lectura comprensiva, Revista Mexicana de Psicología, 23(2), 149-172.

Ibáñez, C. \& Reyes, M.A. (2002). El papel del objeto referente del discurso didáctico en la adquisición de competencias contextuales, Revista Mexicana de Análisis de la Conducta, 28(2), 145-156.

Kantor, J., R. (1933). A Survey of the Science of Psychology. Chicago: Principia Press.

Kantor, J., R. (1959). Interbehavioral Psychology. Chicago: Principia Press.

Kerlinger, F. \& Lee, H. (2002). Investigación del comportamiento. México: McGrawHill. 
Mares, G. (1998). Análisis experimental de la relación entre diferentes competencias lingüísticas. Tesis de Maestría, UNAM-Facultad de Psicología.

Mares, G., Guevara, Y. \& Rueda, E. (1996). Modificación de las referencias orales y escritas a través de un entrenamiento en lectura. Revista Interamericana de Psicología, 30(2), 189-207.

Mares, G., Ribes, E. \& Rueda, E. (1993). El nivel de funcionalidad en lectura y su efecto sobre la transferencia de lo leído. Revista Sonorense de Psicología, 7, 1, 32-43.

Mares, G., Rivas, O. \& Bazán, A. (2002). Configuración en el modo escrito de competencias desarrolladas en forma oral como efecto del nivel funcional de ejercicio. Revista Mexicana de Análisis de la Conducta, 28(2), 173-202.

Mares, Rueda \& Plancarte, (1994). Implicaciones del nivel funcional de la lectoescritura. Revista Integración, (6), 118-123.

Morales, G., Canales, C., Arroyo, R., Pichardo, A., Silva, H. \& Carpio, C. (2005). Efectos del entrenamiento en la identificación de criterios de ajuste lector en estudiantes universitarios. Revista Enseñanza e Investigación en Psicología, 10(2), 239-252.

Moreno, D., Cepeda, L., Tena, O., Hickman, H. \& Plancarte, P. (2005). Conducta gobernada por reglas: Implicaciones educativas. En: C. Carpio \& J. J. Irigoyen (Comps). Psicología y Educación. Aportaciones desde teoría de la conducta (pp. 175-212). México, UNAM FES Iztacala.

Ribes, E. \& López, F. (1985) Teoría de la Conducta: Un modelo de campo y paramétrico. México: Trillas.

Ribes, E., Moreno, R. y Padilla, A. (1996). Un análisis funcional de la práctica científica: Extensiones de un modelo psicológico. Acta Comportamentalia, 4, 205-235.

Sidman, M. (1986). Functional analysis de emergent verbal classes. In: T. Thompson \& M. D. Zeiler (Eds.) Analysis and Integration of Behavioral Units. Hillsdale, New Jersey: Lawrence Erlbaum.

Skinner, B. F. (1938). The behavior of organisms: An experimental analysis. New York: Appleton-century-crofts. 